\title{
A Review of Safety Data from Spontaneous Reports on Marketed Products Containing Tramadol and Celecoxib: A Vigibase Descriptive Analysis
}

Vaqué $A^{1}$, Sust $M^{2}$, Gascón $N^{3}$, Puyada $A^{4}$ and Videla $S^{5 *}$

${ }^{1}$ Drug Safety and Pharmacovigilance. Laboratorios del Dr. Esteve, Barcelona, Spain

${ }^{2}$ Clinical Investigation, Laboratorios del Dr. Esteve, Barcelona, Spain

${ }^{3}$ Drug Safety and Pharmacovigilance. Laboratorios del Dr. Esteve, Barcelona, Spain

${ }^{4}$ Clinical Investigation, Laboratorios del Dr. Esteve, Barcelona, Spain

${ }^{5}$ Clinical Investigation, Laboratorios del Dr. Esteve, Barcelona, Spain

\begin{abstract}
Background: The concomitant administration of opioids and non-steroidal anti-inflammatory drugs is used to manage pain in clinical practice, given their synergistic analgesic effect. Among their possible combinations, tramadol and celecoxib are routinely used. The aim of this study was to explore the safety profile of tramadol and celecoxib administered individually compared to their concomitant administration in clinical practice.

Methods: Retrospective analysis of adverse-drug-reactions from the safety database Vigibase, The WHO global individual case safety report database system.

A case was defined as an adverse-drug-reaction included in a report of Vigibase between January 2000 and March 2012. Three groups were studied: 'tramadol-no-celecoxib' (tramadol was only reported as suspected or interacting drug), 'celecoxib-no-tramadol' (celecoxib was only reported as suspected or interacting drug) and 'celecoxib+tramadol' (both drugs co-administered and reported as suspected or interacting drug). MedDRA dictionary was used to code adverse-drug-reactions. Reporting proportions were calculated as the number of adverse-drugreactions of a given type divided by the overall total number of reported adverse-drug-reaction in each drug-group.

Results: Reporting proportions for global profile, and for each studied group of adverse-drug-reaction, were lower for the concomitant administration than for each individual drug, specifically for the drug (either tramadol or celecoxib) primary involved in the particular adverse-drug-reaction. Therefore, no safety signals were found for 'gastrointestinal bleeding' and 'gastrointestinal signs and symptoms'; 'cardiovascular' and 'cerebrovascular events' (related to 'ischemic and embolic-thrombotic events'); 'renal' and 'renovascular' events (including cardiac failure related events); neither for 'central nervous system' effects; neither for 'respiratory depression'; 'development of tolerance with repeated administration' (including abuse/dependence/withdrawal reported events); 'hepatic disorders (drug-related)'; 'skin events'; and neither for the most frequent preferred terms: 'nausea', 'vomiting', 'constipation', 'myocardial infarction' and 'hypertension'.
\end{abstract}

Conclusion: Based on reporting proportions, no trend was observed to an increased risk for any specific potential safety concern when both tramadol and celecoxib, are administered concomitantly.

Keywords: Tramadol and celecoxib concomitant administration; Safety profile; Adverse drug reaction

List of Abbreviations: ADR: Adverse Drug Reaction; CNS: Central Nervous System; GI: Gastrointestinal; HLGT: High Level Group Terms; HLT: High Level Terms; ICD: International Classification of Diseases; ICH: International Conference on Harmonisation; ICSR: Individual Case Safety Report; LLT: Lowest Level Terms; MedDRA: Medical Dictionary for Regulatory Activities; PT: Preferred Terms; PUB: GI Upper Bleeding; RP: Reporting Proportion; SMQ: Standardized MedDRA Queries; SOC: System Organ Classes; WHO: World Health Organization; WHO-ART: WHO Adverse Reaction Terminology; WHO-DD, WHO-DDE: WHO Drug Dictionaries and the medical terminologies

\section{Introduction}

Pain is the most common symptom for which patients seek medical attention. Currently, in developing countries in particular, a number of serious diseases can cause severe pain, but often little or no pain relief is available [1]. Strategies to address this unmet need include the concomitant administration of analgesic drugs. In fact, in clinical practice is a usual procedure to administer different painkillers at the same time to control the pain. This practice is based on the concept of multimodal analgesia, which is rapidly becoming the 'standard of care'.

Multimodal analgesia involves the use of different classes of analgesics and sometimes different sites of analgesic administration, with the ultimate goal of providing superior pain relief [2-4]. Among the different strategies in multimodal analgesia to manage the pain, the concomitant administration of opioids and non-steroidal antiinflammatory drugs is one of the most used given their analgesic effect [5], being second step therapies in The WHO Pain Relief Ladder [6,7].

*Corresponding author: Videla S, Clinical Investigation, Laboratorios del Dr. Esteve, Barcelona, Spain, Tel: 349-344-660-00; E-mail: svidela@esteve.es

Received August 14, 2014; Accepted October 08, 2014; Published October 10 , 2014

Citation: Vaqué A, Sust M, Gascón N, Puyada A, Videla S (2014) A Review of Safety Data from Spontaneous Reports on Marketed Products Containing Tramadol and Celecoxib: A Vigibase Descriptive Analysis. Adv Pharmacoepidemiol Drug Saf 3: 164. doi:10.4172/2167-1052.1000164

Copyright: @ 2014 Vaqué A, et al. This is an open-access article distributed under the terms of the Creative Commons Attribution License, which permits unrestricted use, distribution, and reproduction in any medium, provided the original author and source are credited. 
The concomitant administration of tramadol and celecoxib is one of the approaches used in clinical practice (data from internal survey based on automated health care databases). Tramadol acts centrally as a weak $\mu$-opioid receptor agonist and an inhibitor of the neuronal reuptake of norepinephrine and serotonin, and celecoxib as an inhibitor of the cyclo-oxygenase-2 enzyme. Currently, tramadol is indicated for the treatment of moderate to severe pain [8], and celecoxib for the relief of chronic inflammatory pain as of osteoarthritis, rheumatoid arthritis and ankylosing spondylitis [9].

Although, up to date, no detectable unexpected safety signals from the free concomitant administration of tramadol and celecoxib (neither in clinical trial data nor post-marketing surveillance data) has been identified; to our knowledge, specific epidemiological studies focused on the safety profile of this concomitant administration of analgesics are not available. An exploratory evaluation of the safety profile of concomitant free administration can be conducted in global safety databases, e.g., Vigibase from the World Health Organization (WHO). Global safety databases record Adverse Drug Reactions (ADR) in terms of Individual Case Safety Report (ICSR), format and content for the reporting of one or several suspected adverse reactions in relation to a medicinal product that occur in a single patient at a specific point of time.

Our hypothesis is that tramadol and celecoxib given concomitantly have a similar safety profile compared to the individual administration of both compounds (at similar doses). Therefore, the aim of this study was to explore the safety profile of tramadol and celecoxib administered individually compared to their free concomitant administration in clinical practice, based on the spontaneous ADR recorded in Vigibase, the WHO Global ICSR Database System.

\section{Methods}

\section{Study design}

This study was a retrospective analysis of ADRs from the global safety database Vigibase. This study was performed according to the stipulations of the Declaration of Helsinki and the level of protection of confidentiality concerning the protection of personal data as required by Spanish laws (LOPD 15/1999) was ensured.

\section{Spontaneous adverse drug reactions}

A case was defined as an ADR included in a report of Vigibase database in which product/s causality was defined as follows:

a) Group 'tramadol no celecoxib', a single drug reported: tramadol is reported as suspected or interacting drug and celecoxib is not present.

b) Group 'celecoxib no tramadol', a single drug reported: celecoxib is reported as suspected or interacting drug and tramadol is not present.

c) Group 'celecoxib+tramadol', both drugs concomitantlyadministered (co-administered) and reported, including:

c.1) tramadol and celecoxib are both reported as suspected or interacting drugs,

c.2) tramadol is reported as suspected or interacting drug and celecoxib is reported as concomitant drug,

c.3) celecoxib is reported as suspected or interacting drug and tramadol is reported as concomitant drug.

The five above mentioned groups (a, b, c.1, c.2 and c.3) are mutually exclusive and do no overlap, i.e., no ADR is contained in more the one group. Likewise, it is worth mentioning that cases in which tramadol and celecoxib are co-administered but both appear reported merely as concomitant drug were not included.

\section{Vigibase}

The main aim of the WHO International Drug Monitoring Programme, started in 1968 , is to identify the earliest possible pharmacovigilance signals. The data held is collected from countries participating in the WHO Medicines Safety Programme. As of May 2012, 107 countries had joined the WHO Medicines Safety Programme. VigiBase is maintained and developed on behalf of the WHO by the Uppsala Monitoring Centre, situated in Uppsala, Sweden. The database system includes the International Conference on Harmonisation of Technical Requirements for Registration of Pharmaceuticals for Human Use (ICH) E2B compatible ICSR database, the WHO Drug Dictionaries (WHO-DD and WHO-DDE), and the medical terminologies WHO Adverse Reaction Terminology (WHO-ART), International Classification of Diseases (ICD), and the Medical Dictionary for Regulatory Activities (MedDRA). VigiBase is used directly by the national centers and is accessed indirectly by other regulatory bodies, the pharmaceutical industry, and academia through data requests to the Uppsala Monitoring Centre. VigiBase contains more than 8,000,000 ICSRs, mainly from Europe and North America [10,11].

Although VigiBase is primarily intended to be a spontaneous ADRs report system, the database includes cases with a varying degree of suspectedness, both on the level of the initial reporter, and on the causality ascertainment made by the national center. Case reports from studies or special monitoring are also included, when provided to the Uppsala Monitoring Centre. Each ICSR can contain more than one $\mathrm{ADR}[10,11]$. It is worth mentioning that, generally, the spontaneous $\mathrm{ADR}$ are recorded at the approved therapeutic doses.

To carry out this study, the following search was requested to Vigibase, with output format 'Summary by Year' and coded using MedDRA terminology: all ADRs for users of single drugs and concomitant administration of tramadol and celecoxib as well as for tramadol and celecoxib co-users. That is to say, we requested a separate search for each of the above mentioned five conditions: $a, b, c .1, c .2$ and c.3.

In order to avoid bias in the comparisons, we focused on the time window when both drugs were marketed. Though the earliest ADRs for tramadol were reported back in 1983 and for celecoxib in 1999, reports in which both are present in the market do not start until 2000. Consequently, this was the starting point, and all reports from January 2000 up to March 2012 were included in the analysis.

Vigibase facilitated us the requested information in an Excel file in March 2012, which was used for the analysis of this study. The following data were gathered: report ID (Vigibase), year, safety report ID, company, MedDRA SOC, PT and LLT Names.

\section{Medical Dictionary for Regulatory Activities (MedDRA)}

MedDRA is an international medical terminology dictionary (and thesaurus) was developed under the auspices of ICH and maintained by maintenance and support services organization. It is an international medical dictionary applicable to all phases of biopharmaceutical and medical product development. Therefore, it is used by regulatory authorities in the pharmaceutical industry during the regulatory process, from pre-marketing to post-marketing activities, and for data entry, retrieval, evaluation, and presentation. In addition, it is the adverse event classification dictionary endorsed by ICH [12]. 


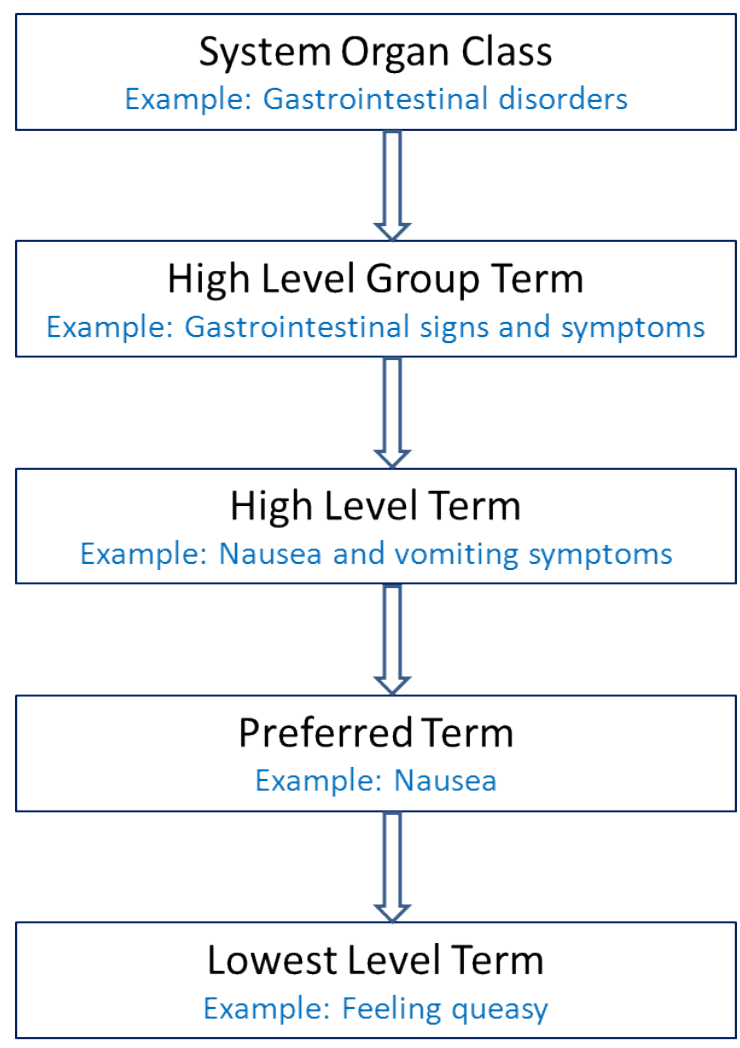

Figure 1: MedDRA hierarchy example.

The MedDRA dictionary is organized in five levels of hierarchy which are, from most specific to most global, as follows: 'Lowest Level Terms' (LLTs) are terms which parallel how information is communicated. These LLTs reflect how an observation might be reported in practice. This level directly supports assigning MedDRA terms within a user database; each member of the next level, 'Preferred Terms' (PTs), is a distinct descriptor (single medical concept) for a symptom, sign, disease diagnosis, therapeutic indication, investigation, surgical or medical procedure, etc. Each LLT is linked to only one PT. Each PT has at least one LLT (itself) as well as synonyms and lexical variants (e.g., abbreviations, different word order). Related PTs are grouped together into 'High Level Terms' (HLTs) based upon anatomy, pathology, physiology, etiology or function. HLTs, related to each other by anatomy, pathology, physiology, etiology or function, are in turn linked to 'High Level Group Terms' (HLGTs). Finally, HLGTs are grouped into 'System Organ Classes' (SOCs) which are groupings by etiology (e.g. Infections and infestations), manifestation site (e.g. Gastrointestinal (GI) disorders) or purpose (e.g. Surgical and medical procedures) [12]. Figure 1 shows the MedDRA hierarchy example.

Standardized MedDRA Queries (SMQs) are used to support signal detection and monitoring. SMQs are validated, standard sets of MedDRA terms that define a search in the database. SMQs include narrow and/or broad terms. Narrow terms are those that are highly likely to represent the condition of interest. For example, the PTs 'pancreatitis acute' and 'pancreatitis haemorrhagic' are narrow terms for the SMQ 'acute pancreatitis' whereas PT 'blood bilirubin' increased is a broad term because not all instances of increased blood bilirubin are indicative of acute pancreatitis [12]. The MedDRA dictionary, version 15.0 , was used in this study.

\section{Statistical analysis}

The sample size was defined as all ADR contained in the WHO database complying our request specifications in the time period of interest.

Three consecutive types of descriptive analyses were performed for tramadol and celecoxib users in comparison to co-users of tramadol and celecoxib:

First analysis-Spontaneous ADR by SOC: ADRs were grouped by primary SOC according to the MedDRA dictionary.

Second analysis: Spontaneous ADR of 'Special Interest' based on known safety profiles of both drugs. Spontaneous ADR of 'Special Interest' was defined in agreement with the safety profile included in the summary of product characteristics of both drugs (as given in the ref 8 and 9 in the section 'Special warnings and precautions for use' and the section 'Undesirable effects' very frequent or frequent). The analysis was performed based on SMQ or other ad hoc grouping considerations as follows:

a) GI bleeding and GI signs and symptoms, which were reviewed and grouped by PT as follows:

a.1) GI upper bleeding (ad hoc grouping): 'GI perforation', 'GI ulceration' and 'GI hemorrhage or bleeding' (PUB).

a.2) GI nonspecific inflammation-dysfunction (SMQ): 'GI nonspecific dysfunction' and 'GI nonspecific inflammation'.

a.3) GI nonspecific symptoms (SMQ): 'GI nonspecific symptoms and therapeutic procedures'.

b) Cardiovascular and Cerebrovascular events, related to 'ischemic and embolic-thrombotic events', were reviewed and grouped by PT as follows:

b.1) Cerebrovascular events (SMQ): 'Ischemic cerebrovascular conditions', 'conditions associated with central nervous system hemorrhages and cerebrovascular accidents'.

b.2) Embolic and thrombotic events (SMQ): 'Embolic and thrombotic arterial events', 'embolic and thrombotic venous events' and 'embolic and thrombotic vessel type unspecified and mixed arterial and venous events'.

b.3) Cardiovascular events (SMQ): 'Myocardial infarction' and 'other ischemic heart disease'.

c) Renal and Renovascular events, including cardiac failure related events, were reviewed and grouped by PT as follows (SMQ): 'Acute renal failure,' 'cardiac failure' and 'renovascular disorders'.

d) Central nervous system (CNS) effects: some concrete PTs from the Nervous System Disorders SOC were selected based on the highest reporting frequency in the postmarketing surveillance ( $a d$ hoc grouping): 'dizziness, 'headache' and 'somnolence'. Another PT, 'fatigue', PT, from the 'General Disorders and Administration Side Conditions' SOC was additionally included.

e) Respiratory depression, and in absence of an appropriate SMQ, the following HLTs were reviewed: 'Breathing abnormalities' and 'respiratory failures' (excluding neonatal).

f) Development of tolerance with repeated administration, including abuse/dependence/withdrawal reported events, were reviewed and grouped by PT as follows ( $a d$ hoc grouping): 'Drug abuse' and 'drug withdrawal'. 


\section{ADR profile by SOC. \% over total reported ADRs by drug exposure}

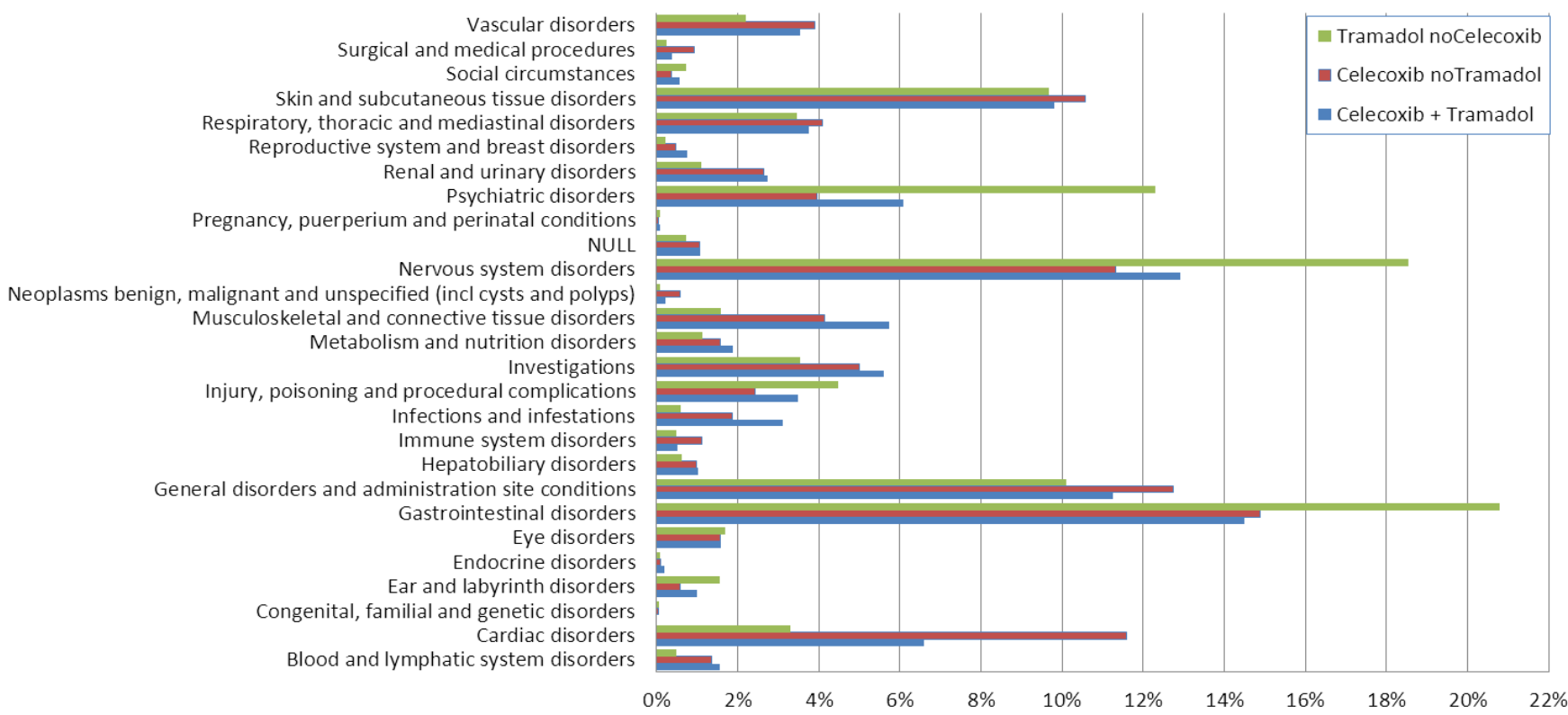

NULL: corresponds to ADRs which, for any reason, have not been assigned a MedDRA code in the Vigibase database

Figure 2: Reporting Proportions per 100 Adverse Drug Reactions by System Organ Class and drug-group.

g) Hepatic disorders (drug-related), were reviewed and grouped by PT as Hepatic disorders (drug-related) (ad hoc grouping): 'Cholestasis and jaundice of hepatic origin', 'hepatic failure', 'fibrosis' and 'cirrhosis and other liver damage-related conditions' and 'hepatitis, noninfectious'.

h) Skin events, the most clinically relevant Skin events, were reviewed and grouped by PT as follows (SMQ): 'Severe cutaneous adverse reactions'

i) The most frequent PTs (based on ADRs expected in both SPCs, as given in the references 8 and 9 in the section 'Undesirable effects'), following the specific PTs were analyzed (ad hoc grouping): 'Nausea', 'vomiting', 'constipation', 'myocardial infarction' and 'hypertension'.

Third analysis-Spontaneous ADR of Special Interest but based on observed data: A data-driven analysis was performed to further evaluate any other potential safety concern different from those detailed above and raised from the free-use of the concomitant administration of tramadol and celecoxib. SOCs showing a higher reporting proportion for 'celecoxib+tramadol' group than for both individual drug-groups were to be explored more in depth, selecting the most reported PTs.

Reporting Proportion (RP): The frequency of reporting of ADRs to Vigibase, among users of the drugs of interest, was described through the calculation of the RP as a summary measure. The RP was defined as the proportion of ADRs (as of PTs) that meet the definition of 'special interest', among all ADRs of a given drug exposure group (drug-group), i.e. 'number of ADRs with the definition of special Interest/total number of reported ADRs'.

For the first analysis, RPs were calculated as the number of ADRs that belong to a given SOC divided by the overall total number of reported ADRs in each drug-group. For the second analysis, RPs were calculated as the number of ADRs that belong to a given grouping (i.e., SMQ or HLT) divided by the overall total number of reported ADRs in each drug-group. It is important to note that PTs included in more than one SMQ grouping were counted in the calculation of the RP of each of these SMQ groupings. For the third analysis, the RP were calculated as the number of ADRs (single PT) divided by the total number of reported ADRs in the specific SOC for each drug-group. All these analysis focused on the comparison between the RP for the concomitant administration vs. the RP of the 'leading' drug-group primary involved in each particular ADR (marked with an asterisk in tables 1-4).

\section{Results}

\section{General characteristics of the spontaneous ADR set}

A total of 160,938 ADRs were recorded in the VigiBase database, in which either tramadol or celecoxib administered alone or in a concomitant administration are involved as 'suspected' during the study period. Among them, 2,123 ADRs contained both drugs used concomitantly and reported, at least one of them, as suspected drug; 107,545 contained only 'celecoxib no Tramadol' and 51,270 'Tramadol no Celecoxib'

\section{Spontaneous ADR findings by System Organ Class}

Figure 2 depicts the safety profile of the three defined drug-groups in terms of RPs. These RPs reflect the relative weight of each SOC among all ADRs reported for a given drug-group.

\section{Spontaneous ADR findings of special interest, based on the} known safety profiles of both drugs

No detectable safety signals were found. The number of reported ADRs and RPs (per 100 ADRs) concerning GI upper bleeding (PUB), 


\begin{tabular}{|c|c|c|c|c|c|c|c|}
\hline \multirow[b]{2}{*}{ Drug-group } & \multirow[b]{2}{*}{ Total reported } & \multicolumn{2}{|c|}{$\begin{array}{l}\text { Gl upper bleeding } \\
\text { (PUB) }\end{array}$} & \multicolumn{2}{|c|}{$\begin{array}{c}\text { GI non-specific inflammation and } \\
\text { dysfunction }\end{array}$} & \multicolumn{2}{|c|}{ GI non-specific symptoms } \\
\hline & & $\begin{array}{l}\text { (narrow) } \\
\text { Count (\%) }\end{array}$ & $\begin{array}{l}\text { (broad) } \\
\text { Count (\%) }\end{array}$ & $\begin{array}{l}\text { (narrow) } \\
\text { Count }(\%)\end{array}$ & $\begin{array}{l}\text { (broad) } \\
\text { Count (\%) }\end{array}$ & $\begin{array}{l}\text { (narrow) } \\
\text { Count }(\%)\end{array}$ & $\begin{array}{l}\text { (broad) } \\
\text { Count (\%) }\end{array}$ \\
\hline Celecoxib+Tramadol & 2123 & $63(3.0 \%)$ & $79(3.7 \%)$ & $24(1.1 \%)$ & $24(1.1 \%)$ & $156(7.4 \%)$ & $172(8.1 \%)$ \\
\hline Celecoxib noTramadol & 107545 & $3931(3.7 \%)^{*}$ & $5320(5.0 \%)^{*}$ & $1719(1.6 \%)^{*}$ & $1719(1.6 \%)^{*}$ & $6683(6.2 \%)$ & $7730(7.2 \%)$ \\
\hline Tramadol noCelecoxib & 51270 & $122(0.2 \%)$ & $220(0.4 \%)$ & $148(0.3 \%)$ & $148(0.3 \%)$ & $9385(18.3 \%)^{*}$ & $9691(18.9 \%)^{\star}$ \\
\hline Total & 160938 & $4116(2.6 \%)$ & $5619(3.5 \%)$ & $1891(1.2 \%)$ & $1891(1.2 \%)$ & $16224(10.1 \%)$ & $17593(10.9 \%)$ \\
\hline
\end{tabular}

Table 1: Number of reported adverse drug reactions and reporting proportions (per 100 adverse drug reactions) of gastrointestinal bleeding (GI, PUB: Perforation, Ulceration, Bleeding) and GI signs and symptoms (* 'leading' drug-group involved in the ADR).

\begin{tabular}{|c|c|c|c|c|c|c|}
\hline \multirow[b]{2}{*}{ Drug-group } & \multirow[b]{2}{*}{ Total reported } & \multicolumn{2}{|c|}{ Cardiovascular events } & \multicolumn{2}{|c|}{ Cerebrovascular events } & \multirow{2}{*}{$\begin{array}{c}\text { Embolic-thrombotic events } \\
\text { Count (\%) }\end{array}$} \\
\hline & & $\begin{array}{l}\text { (narrow) } \\
\text { Count }(\%)\end{array}$ & $\begin{array}{c}\text { (broad) } \\
\text { Count (\%) }\end{array}$ & $\begin{array}{c}\text { (narrow) } \\
\text { Count }(\%)\end{array}$ & $\begin{array}{c}\text { (broad) } \\
\text { Count (\%) }\end{array}$ & \\
\hline Celecoxib+Tramadol & 2123 & $72(3.4 \%)$ & $78(3.7 \%)$ & $34(1.6 \%)$ & $39(1.8 \%)$ & $107(5.0 \%)$ \\
\hline Celecoxib noTramadol & 107545 & $6809(6.3 \%)^{*}$ & $6922(6.4 \%)^{*}$ & $4715(4.4 \%)^{*}$ & $5057(4.7 \%)^{\star}$ & $12946(12.0 \%)^{*}$ \\
\hline Tramadol noCelecoxib & 51270 & $136(0.3 \%)$ & $200(0.4 \%)$ & $83(0.2 \%)$ & $244(0.5 \%)$ & $259(0.5 \%)$ \\
\hline Total & 160938 & $7017(4.4 \%)$ & $7200(4.5 \%)$ & $4832(3.0 \%)$ & $5340(3.3 \%)$ & $13312(8.3 \%)$ \\
\hline
\end{tabular}

Table 2: Number of reported adverse drug reactions and reporting proportions (per 100 adverse drug reactions) of cardiovascular and cerebrovascular events (* 'leading' drug-group involved in the ADR).

\begin{tabular}{|c|c|c|c|c|}
\hline Drug-group & Total reported & $\begin{array}{c}\text { Dizziness } \\
\text { Count (\%) }\end{array}$ & $\begin{array}{c}\text { Fatigue } \\
\text { Count (\%) }\end{array}$ & $\begin{array}{c}\text { Seadache } \\
\text { Count (\%) }\end{array}$ \\
\hline Colecoxib+Tramadol & 2123 & $51(2.4 \%)$ & $18(0.9 \%)$ & $21(1.0 \%)$ \\
\hline Celecoxib noTramadol & 107545 & $1294(1.2 \%)$ & $761(0.7 \%)$ & $1028(1.0 \%)$ \\
\hline Tramadol noCelecoxib & 51270 & $2252(4.4 \%)^{*}$ & $300(0.6 \%)^{\star}$ & $779(1.5 \%)^{*}$ \\
\hline Total & 160938 & $3597(2.2 \%)$ & $1079(0.7 \%)$ & $209(0.2 \%)$ \\
\hline
\end{tabular}

PT from the SOC of General Disorders and Administration Side Condition

Table 3: Number of reported adverse drug reactions and reporting proportions (per 100 adverse drug reactions) concerning central nervous system effects (* 'leading' drug-group involved in the ADR).

\begin{tabular}{|c|c|c|c|c|c|c|}
\hline Drug-group & Total reported & $\begin{array}{l}\text { Constipation } \\
\text { Count (\%) }\end{array}$ & $\begin{array}{l}\text { Hypertension } \\
\text { Count (\%) }\end{array}$ & $\begin{array}{c}\text { Myocardial infarction } \\
\text { Count (\%) }\end{array}$ & $\begin{array}{l}\text { Nausea } \\
\text { Count (\%) }\end{array}$ & $\begin{array}{l}\text { Vomiting } \\
\text { Count (\%) }\end{array}$ \\
\hline Celecoxib+Tramadol & 2123 & $11(0.5 \%)$ & $16(0.8 \%)$ & $45(2.1 \%)$ & $50(2.4 \%)$ & $30(1.4 \%)$ \\
\hline Celecoxib noTramadol & 107545 & $307(0.3 \%)$ & $1144(1.1 \%)^{\star}$ & $5597(5.2 \%)^{*}$ & $1375(1.3 \%)$ & $822(0.8 \%)$ \\
\hline Tramadol noCelecoxib & 51270 & $288(0.6 \%)^{*}$ & $211(0.4 \%)$ & $54(0.1 \%)$ & $4616(9.0 \%)^{*}$ & $3698(7.2 \%)^{\star}$ \\
\hline Total & 160938 & $606(0.4 \%)$ & $1371(0.9 \%)$ & $5696(3.5 \%)$ & $6041(3.8 \%)$ & $4550(2.8 \%)$ \\
\hline
\end{tabular}

Table 4: Number of reported Adverse Drug Reactions and Reporting Proportions (per 100 Adverse Drug Reactions) for the most frequent Preferred Terms, by drug-group ( 'leading' drug-group involved in the ADR).

GI non-specific inflammation and dysfunction and GI signs and symptoms are presented in Table 1. Table 2 shows the number of reported ADRs and RPs (per 100 ADRs) concerning Cerebrovascular and Cardiovascular events, related to 'ischemic and embolic/thrombotic events' and 'other embolic/thrombotic events'. The number of reported ADRs and RPs (per 100 ADRs) concerning CNS effects are shown in table 3, and the most frequent PTs ('nausea', 'vomiting', 'constipation', 'myocardial infarction' and 'hypertension') in table 4. Similar results were found in renal and renovascular events, respiratory depression, development of tolerance with repeated administration, hepatic disorders and skin events (data not shown).

\section{Spontaneous ADR of special interest based on observed data}

After an in-depth revision of the findings from the above results sections, the following SOCs were also analyzed and evaluated in detail: musculoskeletal and connective tissue disorders, investigations, metabolism and nutrition disorders, infections and infestations as well as blood and lymphatic system disorders. No safety signals from the free concomitant administration of both drugs were detected in these specific spontaneous ADR of special interest (data not shown).

\section{Discussion}

To our knowledge, this is the first study that evaluates the safety profile of the concomitant administration use of tramadol and celecoxib, an approach used as multimodal analgesia in clinical practice. No safety signals of this concomitant administration use of tramadol and celecoxib were found. In fact, these findings could be expected. Both active principles, tramadol and celecoxib, have been marketed worldwide for more than 10 years. During this time period, no safety concerns from the concomitant use of both drugs, either in clinical trials data or in post-marketing surveillance data have been raised. Likewise, there is no risk described for the co-administration in any of the labels of the products, neither in the general sections of the summary of product characteristics such as 'Contraindications' and as given in the references 8 and 9 in the section 'Special warnings and special precautions for use' nor in the specific drug-drug interactions as given in the references 8 and 9 in the section 'Interaction with other medicinal products and other forms of interaction' $[8,9,13]$. Furthermore, the current results are also supported by other lines of knowledge available including as described in the summary of product characteristics of both medicinal products, for instance, that based on the described metabolic routes, no clinically relevant pharmacokinetic drug-drug interactions, that may have safety implications, would be expected with the concomitant use of tramadol and celecoxib at their approved doses.

The observed RPs for the concomitant use of tramadol and 
celecoxib were consistent with the safety profile of each individual drug. A consistent safety profile was observed for their concomitant use. RP for global profile (SOC) and for each group of individual ADRs of interest were lower for the concomitant use than for each individual drug, specifically for the main drug (the 'leading': either tramadol or celecoxib) involved in the ADR.

The most frequent reported SOCs for 'tramadol no celecoxib group' were: GI, CNS and psychiatric disorders; and for 'celexoxib no tramadol' group: GI, cardiac and CNS disorders. In all these higher reporting ADRs, the 'celecoxib+tramadol' group presented a lower RPs than the corresponding primary suspected drug for the ADR. Additionally, general disorders and skin and subcutaneous tissue disorders were equally frequent for tramadol, celecoxib and for the concomitant use. The safety profile of the three defined drug groups was also consistent with what is already known referring to the defined safety concerns or to most reported ADRs in each summary of product characteristics $[8,9]$. For example, it was observed that the RP in the group 'tramadol no celecoxib' were higher for constipation, nausea and vomiting. These results have been previously reported $[8,13-15]$. In the group 'celecoxib no tramadol' the RPs were higher for PUB, hypertension and myocardial infarction. In fact, these ADRs were also expected [9,16-18]. Based on these results, it can be mentioned that this concomitant administration of two well-known active principles, tramadol and celecoxib, with complementary mechanisms of action, does not present increased or unpredictable safety concerns. Therefore, it could be assumed that nor pharmacokinetic nor deleterious pharmacodynamic clinically relevant drug-drug interactions have been observed. Moreover, it could be hypothesized that, since the most relevant side effects for these drugs are dose-dependent, a lower dose would result in a better benefit/risk profile.

As expected, the number of ADRs reported involving 'celecoxib+tramadol' is substantially lower than for the individual drugs, probably due to a lower number of users of the concomitant administration. Nevertheless, these data provides an idea on the current use of this co-administration. In this line, more than 7,000 patients have been identified to be exposed to the aforementioned concomitant administration in the UK during the same period using the automated health care database Clinical Practice Research Datalink (internal data). Therefore, all these data provide evidence that this co-administration of analgesic medicinal products (tramadol and celecoxib), is administered in clinical practice to the general population.

The main strength of this study is that it is based on a standard ADR register, The WHO Global ICSR Database System, and that it has been used an international medical dictionary, MedDRA. Nevertheless, there are several limitations to this study which should be considered. Limitations related to spontaneous reporting of ADRs and the interpretation of RP as: i) ADRs are under-reported, being estimated between $50-90 \%$ (i.e., reporting may be more frequent with severe events and less frequent when the effects are well known) $[19,20]$; ii) The channeling of a drug to lower or higher risk patients may alter the occurrence of ADRs (i.e. in oncologic pain, patients receiving free concomitant administration of tramadol and celecoxib are in the second step therapy in The WHO Pain Relief Ladder [6,7], pointing to a potentially more severe patient population, which may lead to higher reporting of ADRs [21]; iii) ADRs identification and reporting rates may be higher if there have been recent warnings about a drug (notoriety bias) or early after marketing authorization and other factors (i.e. extent of use, publicity, etc.) which vary over time, from product to product and country to country $[10,19]$; and iv)
Length of time on the market and familiarity with the drug have been shown to affect reporting rates $[10,19]$. Another limitation is the lack of information about the number of patients exposed to the product, in addition to uncertainties as to the indication for which an analgesic was prescribed. However, in most cases the spontaneous ADR are recorded on regular clinical practice and thus may correspond to the approved therapeutic doses: for tramadol, $200 \mathrm{mg}$ per day is a usual therapeutic dose and for celecoxib, $200 \mathrm{mg}$ per day is a recommended therapeutic dose. Likewise, the data contained per se in Vigibase and the limited details about each suspected ADR may underestimate the results. The reports, which are submitted to National Centers, come from both regulatory and voluntary sources. Some National Centers accept reports only from medical practitioners; other National Centers accept reports from a wider spectrum of health care professionals. Also, processing time varies from country to country. Therefore, reporting figures obtained from the Collaborating Centre may differ from those obtained directly from National Centers. For the above reasons it is clear that the information is not homogeneous at least with respect to origin or likelihood that the pharmaceutical product caused the adverse reaction. Interpretation of ADRs data, and particularly those based on comparisons between pharmaceutical products, may be overestimating or underestimating the results.

In spite of these limitations, spontaneous reporting of ADRs remains one of the most important methods for monitoring the safety of drugs, and the analyses of adverse events databases have demonstrated to be useful for detecting trends and hypothesis generation. Therefore, the results of this study, based on reporting proportions, allow concluding that no trend to an increased risk for any specific potential safety concern when both drugs, tramadol and celecoxib, are administered concomitantly was observed. These findings should be confirmed with other epidemiological studies or with randomized clinical trials.

The information derived from our analysis does not represent the opinion of The WHO [22].

\section{References}

1. IASP $^{\circledR}$ Global Year Against Pain.

2. Buvanendran A, Kroin JS (2009) Multimodal analgesia for controlling acute postoperative pain. Curr Opin Anaesthesiol 22: 588-593.

3. Elvir-Lazo OL, White PF (2010) Postoperative pain management after ambulatory surgery: role of multimodal analgesia. See comment in PubMed Commons below Anesthesiol Clin 28: 217-224

4. Argoff CE, Albrecht P, Irving G, Rice F (2009) Multimodal analgesia for chronic pain: rationale and future directions. Pain Med: S53-S66.

5. Miranda HF, Silva E, Pinardi G (2004) Synergy between the antinociceptive effects of morphine and NSAIDs. Can J Physiol Pharmacol 82: 331-338.

6. World Health Organization (1996). Cancer pain relief: with a guide to opioid availability. In: Geneva: World Health Organization. (2ndedsn), Geneva, Switzerland.

7. World Health Organization Collaborating Center (2006). Appraising the WHO Analgesic Ladder on its 20th anniversary. Cancer Pain Release.

8. Tramadol. Summary of Product Characteristics of Adolonta $50 \mathrm{mg}$ hard capsules, AEMPS web (Spain). (http://www.aemps.gob.es/cima/ especialidad. do?metodoverFichaWordPdf\&codigo=59088\&formato=pdf\&formulario=FICHA S\&file=ficha.pdf)

9. Celebrex 100mg \& 200mg Capsules, emc, UK.

10. Lindquist M (2008) VigiBase, the WHO Global ICSR Database System: Basic Facts. Drug Information Journal 42:409-419.

11. http://www.umc-products.com/

12. http://www.meddra.org/ 
Citation: Vaqué A, Sust M, Gascón N, Puyada A, Videla S (2014) A Review of Safety Data from Spontaneous Reports on Marketed Products Containing Tramadol and Celecoxib: A Vigibase Descriptive Analysis. Adv Pharmacoepidemiol Drug Saf 3: 164. doi:10.4172/2167-1052.1000164

Page 7 of 7

13. Martindale W, Parfitt K (1999) The complete drug reference: Pharmaceutical Press (32ndedn), London UK.

14. Radbruch L, Grond S, Lehmann KA (1996) A risk-benefit assessment of tramadol in the management of pain. Drug Saf 15:8-29.

15. Pergolizzi J, Böger RH, Budd K, Dahan A, Erdine S, et al. (2008) Opioids and the management of chronic severe pain in the elderly: consensus statement of an International Expert Panel with focus on the six clinically most often used World Health Organization Step III opioids (buprenorphine, fentanyl, hydromorphone, methadone, morphine, oxycodone). Pain Pract 8:287-313.

16. Public CHMP Assessment Report for Medicinal products containing NonSelective Non Steroidal Anti-Inflammatory Drugs (NSAIDS). European Medicines Agency.

17. Kearney PM, Baigent C, Godwin J, Halls H, Emberson JR, et al. (2006) Do selective cyclo-oxygenase-2 inhibitors and traditional non-steroidal antiinflammatory drugs increase the risk of atherothrombosis? Meta-analysis of randomised trials. BMJ 332: 1302-1308
18. Castellsague J, Holick CN, Hoffman CC, Gimeno V, Stang MR, et al. (2009) Risk of upper gastrointestinal complications associated with cyclooxygenase-2 selective and nonselective nonsteroidal antiinflammatory drugs. Pharmacotherapy 29: 1397-1407

19. Strom BL, Kimmel SE, Hennessy S (2012) Pharmacoepidemiology. (5th edsn), John Wiley \& Sons, Ltd.

20. Leufkens HG (2000) Pharmacoepidemiology and gastroenterology: a close couple. Scand J Gastroenterol:105-108.

21. de Graaf L, Fabius MA, Diemont WL, van Puijenbroek EP (2003) The Webercurve pitfall: effects of a forced introduction on reporting rates and reported adverse reaction profiles. Pharm World Sci 25: 260-263.

22. CAVEAT DOCUMENT. Accompanying statement to data released from the Uppsala Monitoring Centre, WHO Collaborating Centre for International Drug Monitoring. Uppsala, Sweden. 